
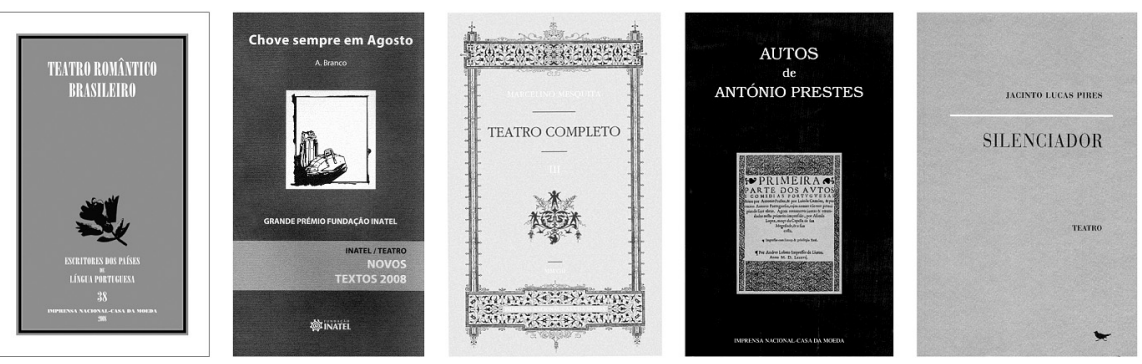

José Maria

Vieira Mendes

\title{
Publicações de teatro em 2008
}

\section{Lista compilada por Sebastiana Fadda}

\section{Peças originais (ou volumes de peças) em primeira edição}

AA.W., Panos: Palcos novos, palavras novas, (Ácido desoxirribonucleico, de Dennis Kelly, trad. Jacinto Lucas Pires; Escudos humanos, de Patrícia Portela; Fim de linha, de Letizia Russo; A vida em Vénus, de Luisa Costa Gomes), Lisboa, Fundação Caixa Geral de Depósitos / Culturgest, 2008.

AA.W., Teatro realista brasileiro (O Rio de Janeiro verso e reverso, de José de Alencar; Quase ministro, de Machado de Assis; Três lágrimas, de Franklin Távora; Caiu o ministério, de França Júnior; A capital federal, de Artur Azevedo), pref. e organização Duarte Ivo Cruz, Lisboa, Imprensa Nacional-Casa da Moeda, Escritores dos Paises de Língua Portuguesa, 2008.

AA.W., Teatro romântico brasileiro (António José ou o poeta e a Inquisição, de Gonçalves de Magalhães; 0 noviço, de Martins Pena; Leonor de Mendonça, de Gonçalves Dias; Macário, de Álvares de Azevedo; A torre em concurso, de Joaquim Manuel de Macedo; Gonzaga, ou a revolução de Minas, de Castro Alves), pref. e organização Duarte Ivo Cruz, Lisboa, Imprensa Nacional-Casa da Moeda, Escritores dos Países de Lingua Portuguesa, 2008.

BRANCO, A., Chove sempre em Agosto, Lisboa, Fundação Inatel, Grande Prémio Fundação Inatel - Novos Textos 2008, 2008.

CALDAS, Miguel Castro, Comida / Casas / Repartição, Lisboa, Artistas Unidos / Culturgest / Teatro de Inverno / Livros Cotovia, Livrinhos de Teatro, 2008.

CARVALHO, António Herculano de, Teatro e poesia (inclui as peças A parede inconstante, "Pode vir a acontecer...", Tesouros), introd. dos filhos do autor (Maria Domitila, António Manuel e Ana Maria), pref. Henrique Barrilaro Ruas, texto de Jorge Calado, Lisboa, IST - Instituto Superior Técnico, 2008.

COUTO, Diogo do, 0 soldado prático, fixação do texto e notas Prof. M. Rodrigues Lapa, Lisboa, Sá da Costa Editora, Clássicos, 2008.

DEL CARLO, Marcantonio, Degraus, pref. Henrique Garcia, Lisboa, SeteCaminhos, 2008.

JONAS, Daniel, Nenhures, Lisboa, Livros Cotovia, Teatro, 2008.
MENDES, Fábio, The Cachorro Manco Show, texto vencedor da 2. edição do Prémio Luso-Brasileiro de Dramaturgia António José da Silva, Lisboa, Instituto Camões, 2008.

MESQUITA, Marcelino, Teatro completo (vol. III: Sinhá, 0 tio Pedro, Uma anedota, A noite do Calvário, D. João III ou o Rei maldito, Almas doentes), org. Duarte Ivo Cruz, Lisboa, Imprensa Nacional-Casa da Moeda, Biblioteca de Autores Portugueses, 2008.

NERY, Júlia, Aquário na gaiola:Drama em dois actos, texto de apresentação de Carlos Avilez, Lisboa, Sextante Editora, 2008

OLIVEIRA, Ana Cristina, Segredos do Levante: Teatro, pref. Maria da Graça A. M. Ventura, introd. da autora, Lisboa, Edições Colibri, 2008.

PAÇOS, Fernando de, Teatro infantil: 15 peças de teatro, pref. António Manuel Couto Viana, Lisboa, Editorial Verbo, 2008.

PEIXOTO, José, Os guardas do Museu de Bagdad, ilustrações de Rui A. Pereira, Porto, Campo das Letras, Campo do Teatro, 2008.

PESSOA, Fernando, O marinheiro, introd. Teresa Rita Lopes, s/l, Livros de Areia, Teatro, 2008.

PIRES, Jacinto Lucas, Silenciador, Lisboa, Livros Cotovia, Teatro, 2008.

PORTELA, Patrícia, Para cima e não para norte, Lisboa, Editorial Caminho, 2008.

PRESTES, António, Autos (contém as peças Auto da Ave Maria, Auto do procurador, Auto do desembargador, Auto dos dous irmãos, Auto da ciosa, Auto do mouro encantado, Auto dos cantarinhos), introd. José Camões, edição de José Camões e Helena Reis Silva, notas de leitura de Eugénio Asensio, Lisboa, Imprensa Nacional-Casa da Moeda, Biblioteca de Autores Portugueses, 2008.

ROSA, Armando Nascimento, Antigona gelada, pref. Maria do Céu Fialho, nota de abertura do autor, Coimbra, Fluir Perene / Centro de Estudos Clássicos e Humanisticos da Universidade de Coimbra, 2008.

SÁ-CARNEIRO, Mário de, Feliz pela infelicidade seguido de 0 vencido, Lisboa, Padrões Culturais Editora, Textos Extraordinários, 2008. 

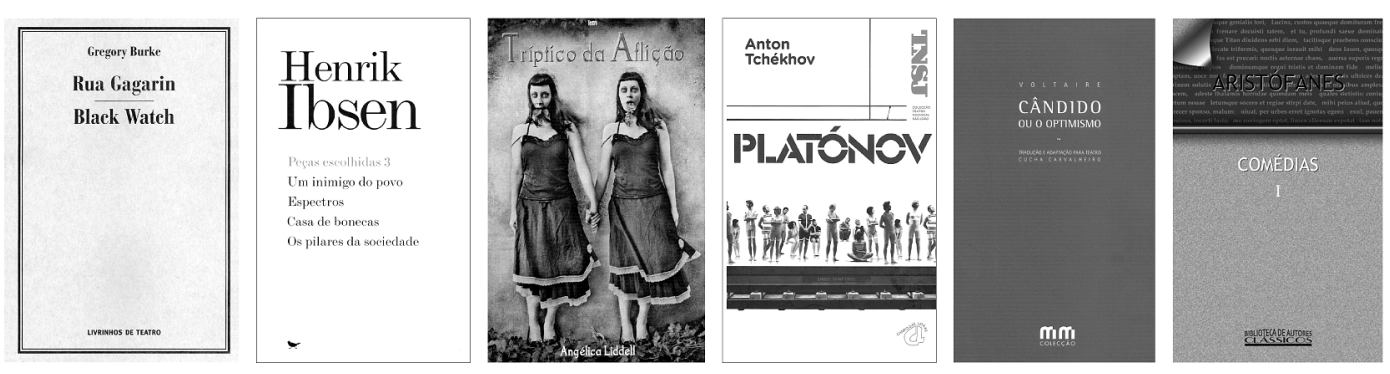

SAMPAIO, Jaime Salazar, Lanterna mágica (peças curtas, curtinhas e encurtadas), pref. António Braz Teixeira, Lisboa, Dimensão 6, 2008.

TELMO, António, A verdade do amor seguido de Adoração. Cânticos de amor de Leonardo Coimbra, Sintra, Zéfiro, 2008.

VIEIRA MENDES, José Maria, Teatro (Dois homens, T1, Se o mundo não fosse assim, A minha mulher, O Avarento ou A última festa, Onde vamos morar, Aos peixes), Lisboa, Livros Cotovia, Teatro, 2008.

\section{Traduções}

ANAGNOSTAKI, Lula, A cidade: Uma trilogia, trad. José António Costa Ideias, Lisboa, Artistas Unidos / Livros Cotovia, Livrinhos de Teatro, 2008.

ARISTÓFANES, As aves, trad. do grego, introd. e notas de Maria de Fátima Sousa Silva, Lisboa, Edições 70, Clássicos Gregos \& Latinos, 2008.

ARISTÓFANES, As rãs, pref., trad. do grego, introd. e notas de Américo Costa Ramalho, Lisboa, Edições 70, Clássicos Gregos \&t Latinos, 2008.

BERNHARD, Thomas, 0 presidente, trad. e introd. de José A. Palma Caetano, s/l, Livros de Areia, Teatro, 2008.

BURKE, Gregory, Rua Gagarin / Black Watch, trad. João da Ponte e João Rosas, Lisboa, Artistas Unidos / Livros Cotovia, Livrinhos de Teatro, 2008.

CORNEILLE, Pierre, Le Cid, pref. e trad. Vasco Graça Moura, edição bilingue, Lisboa, Bertrand Editora, 2008.

CROUCH, Tim, Um carvalho/Inglaterra, uma peça para galerias, trad. Francisco Frazão, Lisboa, Artistas Unidos / Culturgest / Livros Cotovia, Livrinhos de Teatro, 2006.

ENSLER, Eve, Os monólogos da vagina, trad. Isabel Sequeira, Mem Martins, Publicações Europa-América, Contemporânea, 2008.

ÉSQUILO, Oresteia:Agamémnon, Coéforas, Euménides, introd. trad. do grego e notas de Manuel de Oliveira Pulquério Lisboa, Edições 70, Clássicos Gregos \&t Latinos, 2008.

ÉSQUILO, Prometeu agrilhoado, introd., trad. do grego e notas de Ana Paula Quintela Sottomayor, Lisboa, Edições 70, Clássicos Gregos \&t Latinos, 2008.

FOSSE, Jon, Sou o vento/Sono/O homem da guitarra, trad. Pedro Porto Fernandes, Lisboa, Artistas Unidos / Livros Cotovia, Livrinhos de Teatro, 2008.

GOLDONI, Carlo, Peças escolhidas, vol. I ( 0 servidor de dois amos, A estalajadeira, 0 campiello), trad. Alessandra Balsamo (O servidor de dois amos), Jorge Silva Melo ( $A$ estalajadeira) e José Colaço Barreiros (0 campiello), pref. e nota final de Jorge Silva Melo, Lisboa, Livros Cotovia, Teatro, 2008.
IBSEN, Henrik, Peças Escolhidas, vol. III (Um inimigo do povo, Espectros, Casa de boneca, Os pilares da sociedade), trad. Francis Henrich Aubert (Um inimigo do povo), Susana Janic (Espectros), Karl Erik Scholhammer e Aderbal Freire Filho (Casa de boneca) Pedro Fernandes (Os pilares da sociedade), texto em apêndice de Jorge Silva Melo, Lisboa, Livros Cotovia, Teatro, 2008.

LIDDELL, Angélica, Tríptico da aflição (O casal Palavrakis, Onde Upon a Time in West Asphixia, Hysterica Passio Lesões incompativeis com a vida), trad. Alberto Augusto Miranda, Évora, Intensidez, Colecção Teatro, 2008.

LOHER, Dea, Tatuagem / Inocência, trad. José Maria Vieira Mendes, Lisboa, Artistas Unidos / Livros Cotovia, Livrinhos de Teatro, 2008.

MANKELL, Henning, Os antílopes, trad. Solveig Nordlund e Armando Silva Carvalho, com textos de MiguelPedro Quadrio, Solveig Nordlund, Jean-Pierre Vincent e Henning Mankell, s/l, Livros de Areia, Teatro, 2008.

MAYORGA, Juan, 0 rapaz da última fila / Palavra de cão / Bucha e Estica, trad. António Gonçalves, Lisboa, Artistas Unidos / Livros Cotovia, Livrinhos de Teatro, 2008.

MENANDRO, Obra completa (Escudo, Lavrador, Duplo engano, Misantropo, Arbitragem, Herói, Possessa, Cartaginês, Citarista, Adulador, Bebedoras de cicuta Odiento, Mulher de cabelo rapado, Moça de Perinto, Rapariga de Samos, Siciónio, Aparição), trad., introd. e notas de Maria de Fátima Sousa e Silva, Lisboa, Imprensa Nacional-Casa da Moeda, Biblioteca de Autores Clássicos, 2008.

NEILSON, Anthony, O maravilhoso mundo de dissocia / Realismo, trad. Joana Frazão, Lisboa, Artistas Unidos / Livros Cotovia, Livrinhos de Teatro n. ${ }^{\circ} 28,2008$.

SHAKESPEARE, William, Como vos aprouver, trad., introd. e notas de Fátima Vieira, Porto, Campo das Letras, Shakespeare para o século XXI, Obra Dramática Completa, 2008.

SHAKESPEARE, William, 0 mercador de Veneza, trad. Danie Jonas, Lisboa, Livros Cotovia, Teatro, 2008.

SCHILLER, Friedrich Von, Don Carlos, trad. Frederico Lourenço, Lisboa, Livros Cotovia, Teatro, 2008.

SCHILLER, Friedrich Von, Wallenstein: Poema dramático, trad. Maria Hermínia Brandão, Porto, Campo das Letras, Campo do Teatro, 2008.

SÓFOCLES, Rei Édipo, introd., trad. do grego e notas de Maria do Céu Zambujo Fialho, Lisboa, Edições 70, Clássicos Gregos \&t Latinos, 2008.

SONDHEIM, Stephen, Sweeney Todd: 0 terrivel barbeiro de Fleet Street, (libreto de Hugh Wheeler, baseado na 

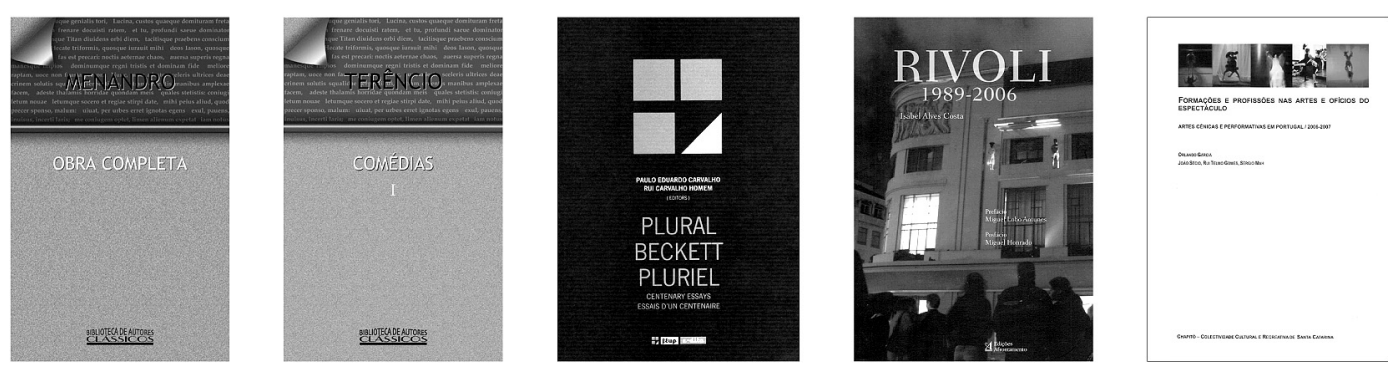

peça Sweeney Todd de Christopher Bond), versão portuguesa de João Lourenço, José Fanha e Vera San Payo de Lemos, Lisboa, Relógio d'Água, 2008.

TCHÉKHOV, Anton, Platónov, trad. António Pescada, Porto, Campo das Letras / Teatro Nacional S. João, Colecção Teatro Nacional S. João, 2008.

TERÊNCIO, Comédias, 2 vols. (vol. I: A moça que veio de Andros, 0 homem que se puniu a si mesmo, Eunuco; vol. II: Formião, A sogra, Os dois irmãos), introd. geral de Walter de Medeiros; trad, introd. e notas de Walter de Medeiros e Aires Pereira do Couto, Lisboa, Imprensa Nacional-Casa da Moeda, Biblioteca de Autores Clássicos, 2008.

VOLTAIRE, Cândido ou o optimismo, tradução e adaptação para teatro de Cucha Carvalheiro, Lisboa, Maria Matos Teatro Municipal / EGEAC, Colecção Maria Matos, 2008.

\section{Traduções em reedição}

WEDEKIND, Frank, 0 despertar da Primavera, trad. Maria Adélia Silva Melo, pref. e apresentação do autor não assinados, Lisboa, Editorial Estampa, (1973, 1. . ed.), 2008, 3. a ed.

\section{Estudos / Documentos}

BORGES, Vera, Teatro, prazer e risco: Retratos sociológicos de actores e encenadores portugueses, Lisboa, Roma Editora, Colecção Evolução, 2008.

BRO0K, Peter, 0 espaço vazio, trad. Rui Lopes, Lisboa, Orfeu Negro, 2008.

CARVALHO, António Carlos, João Villaret: Uma biografia, Lisboa, Ulisseia, Colecção Os Afluentes da Memória, 2008.

CARVALHO, Paulo Eduardo / HOMEM, Rui Carvalho (ed.), Plural Beckett Pluriel: Centenary essays / Essais d'un centenaire, (contém ensaios de Bruno Clément, Rónán McDonald, Steve Wilmer, Maria Helena Serôdio, Hélène Lecossois, Miguel Ramalhete Gomes, Ana Isabel Moniz, Carla Ferreira de Castro, José Domingues de Almeida, Luis Dias Martins, Filomena Vasconcelos, Nadia Louar, Helen Astbury e Paula Seixas) Porto, Faculdade de Letras da Universidade do Porto, 2008.

COSTA, Isabel Alves, Rivoli - 1989-2006, pref. Miguel Lobo Antunes, posfácio Miguel Honrado, nota introdutória da autora, Porto, Edições Afrontamento, 2008.

GARCIA, Orlando et al. (org.), Formações e profissões nas artes e ofícios do espectáculo, Lisboa, Chapitô, 2008.
LOURENÇO, Frederico, Novos ensaios helénicos e alemães, Lisboa, Livros Cotovia, Ensaio, 2008.

MARQUES, Paulo, Amélia Rey Colaço: A imperadora, Lisboa, Parceria A.M. Pereira Livraria Editora / Público, Cadernos Biográficos n. ${ }^{\circ}$ 9, 2008.

--, Beatriz Costa: A miúda da franja: 1907-1996, Lisboa, Parceria A.M. Pereira Livraria Editora / Público, Cadernos Biográficos n. ${ }^{\circ}$ 8, 2008.

--, Mário Viegas: 0 sonho ao poder, Lisboa, Parceria A.M. Pereira Livraria Editora / Público, Cadernos Biográficos n. ${ }^{\circ} 16,2008$

REIS, Luciano, Beatriz Costa, Lisboa, Sete Caminhos, 2008. TRINDADE, Luis, Vasco Santana: Fotobiografias século XX (dir. Joaquim Vieira), Rio de Mouro, Círculo de Leitores e Autores, 2008

\section{Outras reedições}

ROMILLY, Jacqueline de, A tragédia grega, trad. Leonor

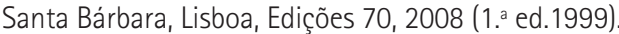

\section{Publicações periódicas}

Artistas Unidos: Revista n. ${ }^{\circ}$ 21-22 (Julho 2008), dir. Jorge Silva Melo, Lisboa, Livros Cotovia.

MACA: Magazine de Arte de Coimbra \&t Afins, n. 1 (MarçoMaio 2008), n. 2 (Junho-Agosto 2008), n. 3 (SetembroNovembro 2008), dir. Ricardo Pinto, Coimbra, Associação Cultural de Música e Teatro à Parte.

Magma n. 6 (["Dramaturgias portuguesas contemporâneas 2", coord. Carlos Alberto Machado, peças de: Jaime Rocha, Quatro cegos; Marcela Costa, A angústia do museólogo (antes da abertura da exposição); Reinaldo Maia e Jorge Louraço Figueira, Cabaré da Santa; Tiago Rodrigues, A partir de amanhã] Junho 2008), dir. Sara Santos e Carlos Alberto Machado, Lages do Pico, Câmara Municipal das Lages do Pico, Culturpico.

Obscena: Revista de artes performativas n. ${ }^{\circ}$ 13-14 (JunhoJulho 2008), n.o 15 (Outubro 2008) e n.'s 16-17 (Novembro-Dezembro 2008), dir. Tiago Bartolomeu Costa, Lisboa.

Sinais de cena n. ${ }^{\circ}$ s. 9 (Junho 2008) e 10 (Dezembro 2008), dir. Maria Helena Serôdio, Associação Portuguesa de Críticos de Teatro \&t Centro de Estudos de Teatro Porto, Campo das Letras. 

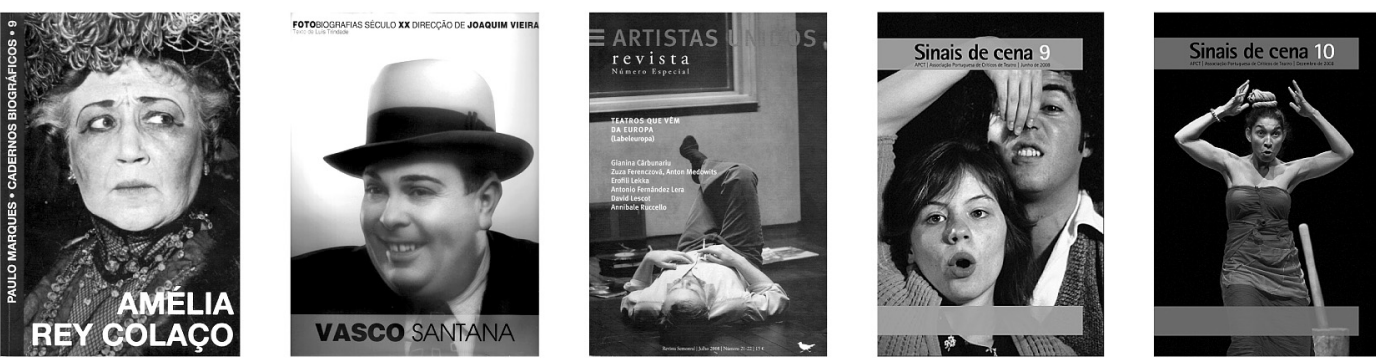

\section{Adenda à lista publicada na Sinais de cena n. ${ }^{\circ} 9$ (2008)}

ARAÚJO, Jorge Cabral, Uma grande tourada: Comédia em dois actos, Lisboa, s/e, 2007. [Peça]

MARNOTO, Rita, Luigi Pirandello e a recepção da sua obra em Portugal, Coimbra, Instituto de Estudos Italianos, Faculdade de Letras da Universidade de Coimbra, Leonardo, 2007. [Estudos / Documentos]

PESSANHA, Vasco Ramirez (coord.), Beatriz Costa: 1907 2007. Álbum de retratos, Lisboa, com textos de José Carlos Alvares e Carlos Alberto Damas, Lisboa, Instituto dos Museus e da Conservação / Centro de Estudos da História do Banco Espírito Santo, 2007. [Estudos / Documentos]

CENTENO, Yvette K., Teatro e sociedade [ler/ver teatro], Lisboa, Edições Universitárias Lusófonas, Colecção de Estudos de Comunicação, Cultura e Tecnologias, 2007. [Estudos / Documentos]

PAVESE, Cesare, Diálogos com Leucò, trad. José Colaço Barreiros, Lisboa, Assírio e Alvim, 2007. [Peça]

VICENTE, Gil, Frágua de amor, Floresta de enganos, trad. dos versos castelhanos José Bento, Lisboa, Assírio \& Alvim, 2007. [Peças]

Magma, n.5 (["Dramaturgias portuguesas contemporâneas 1", coord. Carlos Alberto Machado, texto de: Gonçalo M. Tavares, Possibilidades e ética: que teatro?; peças de: Abel Neves, Provavelmente uma pessoa; Jacinto Lucas Pires, 0 amor de Adalberto Silva Silva; Mário Cabral, 0 jantar; Pedro Eiras, Monólogos: 0 pirata, Simulacros, 0 aquário, Silêncio, Travessia, Despojos, Do fim do mundo] Dezembro 2007), dir. Sara Santos e Carlos Alberto Machado, Câmara Municipal das Lages do Pico, Lages do Pico, Culturpico. [Publicações periódicas]

\section{Adenda à lista publicada na Sinais de cena n. ${ }^{\circ} 7$ (2007)}

AA.W., CITAC 50 anos: Esta danada caixa preta só a murro é que funciona, Coimbra, Imprensa da Universidade de Coimbra, 2006. [Estudos / Documentos]

AA.W., Pina Bausch: Falem-me de amor, Lisboa, Fenda, 2006 (2. ${ }^{\text {a ed.). }}$ [Estudos / Documentos]

ASMUSSEN, Peter, Crime e outras peças [Sangue jovem / Queimados pelo gelo / Quarto com Sol], trad. Ana Campos e Pedro Porto Fernandes, Lisboa, Artistas Unidos / Livros Cotovia, Livrinhos de Teatro, 2006. [Peça]

GARRET, Almeida, Frei Luís de Sousa, Porto, Porto Editora, 2006. [Peça]

MOLIĖRE, D. João ou O banquete de pedra, trad. Nuno Júdice, Porto, Campo das Letras / Teatro Nacional S. João, Colecção Teatro Nacional S. João, 2006. [Peça] PLAUTO, Cásina, pref., trad. do latim, introd. e notas de Aires Pereira do Couto, Lisboa, Edições 70, Clássicos Gregos \&t Latinos, 2006. [Traduções]

\section{Adenda à lista publicada na Sinais de cena n. ${ }^{\circ} 5$ (2006)}

AA.W., Pina Bausch: Falem-me de amor, Lisboa, Fenda, 2005 (1. ${ }^{\text {e ed.). }}$ [Estudos / Documentos]

FUNK, Gabriela, Os agentes da ordem gramatical: Como rindo se aprende a gramática da língua portuguesa. Peça em 3 actos, com um glossário sobre a terminologia linguística, pref. José Esteves Rei, Lisboa, Editores ASA, 2005. [Peça] 